\title{
The Propagation Delay Effect of the Expansion and Inflation of the Universe
}

\author{
Philipp Kornreich ${ }^{1,2}$ \\ ${ }^{1} 1090$ Wien, Austria \\ ${ }^{2}$ King of Prussia, PA, USA \\ Email: pkornrei@syr.edu
}

How to cite this paper: Kornreich, P. (2018) The Propagation Delay Effect of the Expansion and Inflation of the Universe. Journal of Applied Mathematics and Physics, 6, 2024-2031.

https://doi.org/10.4236/jamp.2018.610173

Received: September 19, 2018

Accepted: October 21, 2018

Published: October 24, 2018

Copyright $\odot 2018$ by author and Scientific Research Publishing Inc. This work is licensed under the Creative Commons Attribution International License (CC BY 4.0).

http://creativecommons.org/licenses/by/4.0/

\begin{abstract}
The effect of the propagation delay of gravitational interactions results in a singularity of the normalized acceleration of the radius of a sphere representing the Universe. Stephen Hawking in his Inflation Model also discusses a delay type interaction. This term can be used to model the inflationary rapid expansion of the early Universe. Since the Universe is thought to occupy all of space-time, one cannot define a boundary or radius of the Universe. Therefore, the properties of a sphere in the Universe are analyzed. It is assumed that the Universe will behave similarly to this sphere. This analysis is performed by including the effect of the propagation delay of gravitational interactions in Einstein's equation.
\end{abstract}

\section{Keywords}

Space-Time, Universe, Inflation, Einstein Equation, Momentum Current, Propagation Delay

\section{Introduction}

\subsection{A Model for the Radius of the Universe}

The development of the expansion of the Universe can be derived by investigating the radius of the Universe. But, the General Relativity Theory model [1] actually considers that the Universe occupies all of space-time. Thus, one cannot define a boundary or radius of the Universe. Therefore, there is no such thing as a radius of the Universe. As the name implies, space-time has four dimensions, the three spatial dimensions and time as the fourth dimension. It is observed that most galaxies are moving away from each other [2]. From this, one can infer that the Universe and space-time are expanding. 
One can model the four-dimensional Universe by a two-dimensional surface of a balloon. The surface of the balloon like the Universe, has no boundary or radius. One can spray the balloon with dots that represent the galaxies in the Universe. If the balloon expands, the dots will move away from each other like the galaxies move away from each other in the expanding Universe.

In order to calculate the expansion of the surface of the balloon in two dimensions, one can paint a circular area on the surface of the balloon which has a boundary and a radius. One can calculate the expansion of the radius of this circular surface. Since the balloon's surface behaves similarly as the circular surface, this calculation also describes the expansion of the surface of the balloon.

In order to analyze the problem of the expansion of the Universe, one can define a four-dimensional sphere in this Universe or in space-time [3]. The sphere has a boundary and a conventional radius at a particular time. This is similar to the circular surface on the balloon. Saying "at a particular time" provides the fourth dimension. When the Universe expands, the sphere expands with it. Thus, there is an expanding radius. In this article, when one talks of an expanding "Radius of the Universe", one really means that the radius of the sphere in the Universe is expanding. The Universe will behave similarly as the sphere in the Universe [3].

\subsection{The Model of the Propagation Delay}

There are a lot of misconceptions of the delayed interaction in the descriptions published after about 1950. In earlier publications by H. M. Tetrode [4], J. A. Wheeler and R. Feynman [5], P. A. M. Dirac [6] and A. Lande [7] correctly described the delayed interaction using two terms, a so-called Retarded term and a so-called Advanced term. They did not quite solve the causality problem. But these descriptions gave results that agree with experiments and observations. Models developed after 1950 often only used the Retarded term because it was perceived that Advanced term was not causal and thus gave results in complete disagreement with experiments. A model that shows that all three terms necessary for describing the delayed interaction, the Retarded term, the Advanced term and the Recoil term are causal and give results in agreement with observations is given here.

The gravitational interaction between real galaxies takes time to propagate. The propagation delay effect of the gravitational interaction between galaxies involves three terms, the Retarded term, the Advanced term, and the Recoil term [8].

A gravitational interaction acts currently on one galaxy that was radiated by another galaxy in the past. Thus, this interaction travels over a path from the past position and time to the current position and time. This is the causal Retarded interaction term.

The first galaxy uses the information it obtained from the Retarded interaction to radiate an interaction currently towards the other galaxy that might or might not reach it at its future position and time. Thus, this interaction travels 
over a path from its current position and time to its predicted future position. This is the Advanced interaction term. The Advanced interaction is causal since it involves only current and past information.

The first galaxy radiating the interaction currently experiences a Recoil interaction also currently. The Recoil interaction is equal to the magnitude of the Advanced interaction and opposed to its direction. The Recoil interaction will act on the first galaxy currently, whether or not the Advanced interaction reaches its target.

Only the Retarded and Recoil interaction act on the first galaxy. Thus, since the Retarded and Recoil interactions act on a galaxy currently, the delayed interaction process is causal.

The interactions are radiated and received continually. If one were to interchange future and past in the above description one would obtain the same model. Thus this model symmetric was under time inversion.

In the continuum model used to describe the sphere in the Universe, the interactions are between regions of the Universe rather than galaxies. A rudimentary model of the delayed interaction is between regions in the early universe.

For small delays, the sum of the Past and Recoil interactions divided by the square of the round-trip delay times, can be approximated by the second derivative of the distances in the sphere in the Universe. Since the distances in the sphere in the Universe are proportional to the radius of the sphere in the Universe, one can approximate the second derivative of the distances between regions by the second derivative of the radius of the sphere in the Universe times an adjustable constant parameter. The effect of the delay is small, and can thus be collected in a separate additional term to the main equation under consideration.

The Einstein equation is an equation for the second derivative of the radius of the sphere in the Universe. By adding the delay term to Einstein's equation as described above, one now has two second derivatives of the radius of the sphere in the Universe. The two second derivative terms have opposed signs. When the two second derivatives are equal to each other, their amplitudes must be very large since this difference of the second derivatives is equal to a finite term in Einstein's equation. Thus, the equation for the second derivative or acceleration of the radius of the sphere in the Universe has a singularity. This singularity occurs for the radius when the inflation [9] of the Universe occurred. The double integration of Einstein's equation, including the Singularity, to solve for the Radius results in a step in the size of the Radius as a function of time, (see Figure 1).

After this step of the Radius, as a function of time, the Radius follows an accelerating expansion.

\section{Calculation of the Radius of the Sphere in the Universe}

It is consistent with the model used here to assume, for simplicity, that the Universe is homogeneous and isotropic. Small-scale in homogeneity such as stars and galaxies are also neglected. We here use Einstein's equation from Baez and 


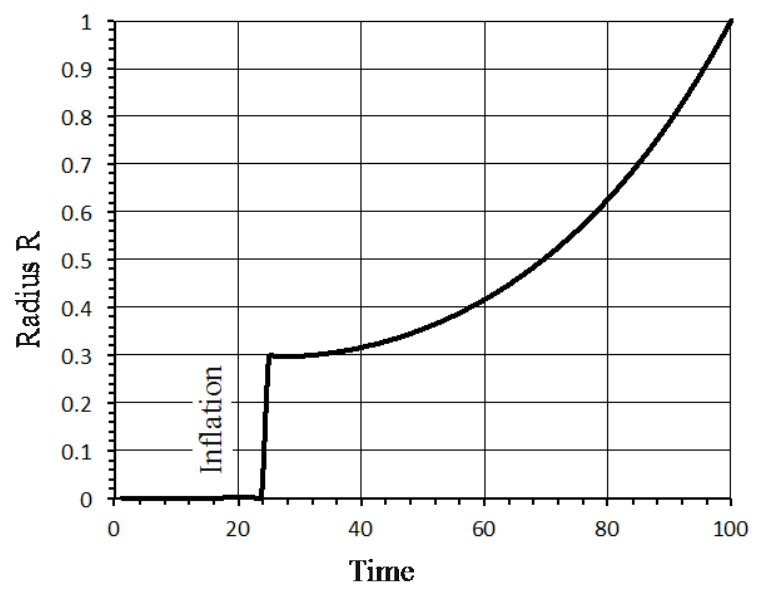

Figure 1. Plot of the normalized radius $\mathrm{R}$ of the sphere in the Universe as a function of time. Note the rapid expansion, or inflation step. The step occurs at the singularity in the denominator of Equation (14). Note, that in this model it is assumed that Einstein's Cosmological Constant is large enough for the radius $\mathrm{R}$ to expand in an accelerating way after the inflationary step.

Bunn [3] instead of deriving it, since the derivation is lengthy. Einstein's Equation which holds in an infinitesimal size ball containing a very large number of even smaller particles at time $t=0$ is given by [3]:

$$
\frac{\ddot{v}}{v}=-\frac{G}{2 c^{2}}\left(\dot{p}_{t}+\dot{p}_{x}+\dot{p}_{y}+\dot{p}_{z}\right)+\frac{c^{2}}{r_{s s}^{2}} \Lambda
$$

where $v$ is the volume, $G$ is the Gravitational constant, $c$ is the speed of light in vacuum and $\dot{p}_{a}$ with $a=t, x, y, z$, is the momentum density current in kg per second squared per meter, as measured at the center of the small ball. Here $\Lambda$ is the normalized, dimensionless Einstein's cosmological constant. All parameters are measured at the center of the small ball in MKS units. The Schwarzschild radius is.

$$
r_{s s}=\frac{2 m G}{c^{2}}
$$

In the equation for the Schwarzschild radius $m$ is the total mass of all the small particles in the above four-dimensional ball. The time component of the momentum current is just the energy density [3].

$$
\dot{p}_{t}=\rho c^{2}
$$

where $\rho$ is the ordinary mass density. Substituting Equation (3) into Equation (1) and assuming the sphere in the Universe is isotropic one obtains at $t=0$ :

$$
\frac{\ddot{v}}{v}=-\frac{G}{2}\left[\rho+\frac{3 \dot{p}}{c^{2}}\right]+\frac{c^{2}}{r_{s s}^{2}} \Lambda
$$

One can model the above sphere in the Universe by a very large number of such infinitesimal spheres. The infinitesimal spheres have a radius $r$. But, since it is assumed that the sphere in the Universe is uniform and isotropic the large sphere with radius $\mathrm{R}$ will behave the same way as the small balls. 


$$
\frac{\ddot{V}}{V}=-\frac{G}{2}\left[\rho+\frac{3 \dot{p}}{c^{2}}\right]+\frac{c^{2}}{R_{s s}^{2}} \Lambda
$$

where $V$ is the volume of the sphere in the Universe. Here the mass $M$ in the Schwarzschild Radius $R_{s s}$ equation is the total mass of the sphere in the Universe. The Volume acceleration divided by the volume $\frac{\ddot{V}}{V}$ can be expressed as follows in terms of the Radius $R$ of the sphere in the Universe:

$$
\text { a) } \frac{\ddot{V}}{V}=3 \frac{\ddot{R}}{R}+6 \frac{\dot{R}^{2}}{R^{2}} \text { at low radial velocities b) } \frac{\ddot{V}}{V} \approx 3 \frac{\ddot{R}}{R}
$$

Substituting Equation (6b) into Equation (5). One obtains for a completely isotropic case:

$$
3 \frac{\ddot{R}}{R}=-\frac{G}{2}\left(\rho+\frac{3 \dot{p}}{c^{2}}\right)+\frac{c^{2}}{R_{s s}^{2}} \Lambda \text { where } \rho+\frac{3 \dot{p}}{c^{2}}>0
$$

The gravitational interaction between real galaxies takes time to propagate. The effect of the propagation delay [8] of the gravitational interaction between galaxies involves the difference in distance $r_{k}-r_{k-1}$ between a current galaxy position at $t_{k}$ and a galaxy position at some previous time $t_{k-1}$. It also involves a Recoil term. When a galaxy radiates a gravitational interaction, it experiences a recoil interaction. The Recoil [4] term is proportional to the magnitude of the difference in distance $r_{k+1}-r_{k}$ between a probable future galaxy position at $t_{k+1}$ and a current galaxy position at current time $t_{k}$ and it is in a direction opposed to that future term. The recoil term will occur whether the radiated term reaches its target or not. Only the Past and Recoil interaction act on a galaxy.

The Universe is modeled as a continuum of more or less uniformly distributed mass. Thus, the delayed interaction describes the interaction between regions of the Universe.

The effect of the delayed interaction is usually very small and it is of the order of the ratio $\frac{R_{s s}}{R}$ of the Schwarzschild radius $R_{s s}$ of the total mass in the sphere in the Universe divided by the radius $R$ of the sphere in the Universe. But in the early Universe apparently, the mass continuum circulated fast enough so that the effect of the delayed interaction became large. Thus, the delayed interaction is important during the very early time of the Universe, before galaxies formed.

Assuming the distances between regions in the Universe is approximately proportional to the radius $R$ of the sphere in the Universe. The effect of the propagation delay can be approximated by two terms. A term describing the action on a Universe region that was radiated by another region in the past $\frac{\rho G}{R} K\left(R_{k}-R_{k-1}\right)$ and a Recoil term $\frac{\rho G}{R} K\left(R_{k+1}-R_{k}\right)$ because this region also radiates an interaction. Indeed, Stephen Hawking [5] also discusses a delay type interaction.

$$
3 \frac{\ddot{R}}{R}=-\frac{G}{2}\left(\rho+\frac{3 \dot{p}}{c^{2}}\right)+\frac{c^{2}}{R_{s s}^{2}} \Lambda+\frac{\rho G}{R}\left[K\left(R_{k+1}-R_{k}\right)-K\left(R_{k}-R_{k-1}\right)\right]
$$

The term in the square bracket represents the effect of the propagation delay. 
$K$ is a proportionality constant. The Recoil term is equal in magnitude and opposed in direction to the Advanced term radiated by a region in the sphere in the Universe. Collecting terms in the square bracket and multiplying and dividing by the square $\tau^{2}=\frac{4 R^{2}}{c^{2}}$ of the round-trip delay time. One should have used the average distance between galaxies in the round-trip delay time. But, it is assumed that the radius $R$ of the sphere in the Universe is proportional to the distances between regions in the sphere in the Universe.

$$
3 \frac{\ddot{R}}{R}=-\frac{G}{2}\left(\rho+\frac{3 \dot{p}}{c^{2}}\right)+\frac{c^{2}}{R_{s s}^{2}} \Lambda+\frac{4 \rho R G}{c^{2}} K \frac{R_{k+1}-2 R_{k}+R_{k-1}}{\tau^{2}}
$$

Approximating the second difference, divided by the square $\tau^{2}$ of the round-trip delay time, by the second derivative one obtains:

$$
3 \frac{\ddot{R}}{R}=-\frac{G}{2}\left(\rho+\frac{3 \dot{p}}{c^{2}}\right)+\frac{c^{2}}{R_{s s}^{2}} \Lambda+\frac{4 \rho R G}{c^{2}} K \ddot{R}
$$

The total Mass $\mathrm{M}$ of the four-dimensional sphere in the Universe is:

$$
\text { a) } M=\frac{4 \pi}{3} R^{3} \rho \quad \text { b) } \rho=\frac{3 M}{4 \pi R^{3}} \text { and c) } R_{s s}=\frac{2 M G}{c^{2}}
$$

Substituting Equation (11b) for the density $\rho$ and the Equation (11c) or the Schwarzschild radius $R_{s s}$ into Equation (10).

$$
3 \frac{\ddot{R}}{R}=-\frac{1}{2}\left(\frac{3 R_{s s} c^{2}}{8 \pi R^{3}}+\frac{3 \dot{p} G}{c^{2}}\right)+\frac{c^{2}}{R_{s s}^{2}} \Lambda+\frac{3 K R_{s s}}{2 \pi R} \frac{\ddot{R}}{R}
$$

Collecting terms

$$
3 \frac{\ddot{R}}{R}\left(1-\frac{K R_{s s}}{2 \pi R}\right)=-\frac{1}{2}\left(\frac{3 R_{s s} c^{2}}{8 \pi R^{3}}+\frac{3 \dot{p} G}{c^{2}}\right)+\frac{c^{2}}{R_{s s}^{2}} \Lambda
$$

Solving for the normalized acceleration $\frac{\ddot{R}}{R}$ of the radius of the sphere in the Universe to obtain this papers final result:

$$
\frac{\ddot{R}}{R}=\frac{-\frac{1}{2}\left(\frac{R_{s s} c^{2}}{8 \pi R^{3}}+\frac{\dot{p} G}{c^{2}}\right)+\frac{c^{2}}{3 R_{s s}^{2}} \Lambda}{1-\frac{K R_{s s}}{2 \pi R}}
$$

Equation (14) is the final result for the normalized acceleration $\frac{\ddot{R}}{R}$ of the radius of the four-dimensional sphere in the Universe. It is a very approximate solution. The numerator of Equation (14) is Einstein's basic equation describing the big bang cosmology of reference [3]. There are two dimensionless adjustable parameters the delay constant $K$ and Einstein's cosmological constant $\Lambda$. A singularity in the acceleration of the radius of the sphere in the Universe occurs at $R=\frac{K R_{s s}}{2 \pi}$, see Figure 1 . The normalized acceleration grows explosively at this value of the radius. This is the Cosmological Inflation [9] [10] [11]. 
The ratio $\frac{R_{s s}}{R}$ of the Schwarzschild radius $R_{s s}$ to the radius $R$ represents the effect of the delayed interaction. Observations point to a rapid growth or Inflation of the early Universe. Thus, the adjustable parameter $K$ must have a substantial value representing some modes in the early gravitational field and mass continuum of the early Universe. But as the radius $R$ of the Universe becomes large, the effect of the delayed interaction decreases substantially. Note from Equations (9) and (10) that the second acceleration term in equation 10 is only an approximation, thus the denominator of Equation (14) does not completely go to zero. In the limit of a very large radius $R$ for a sufficiently large value of Einstein's cosmological constant $\Lambda$, the acceleration of the expansion of the Universe will continue to increase.

\section{Conclusions}

The main contribution of this paper is the addition to Einstein's equation of a term representing the propagation delay of the gravitational interaction between regions in the sphere in the Universe. The resulting equation can be used to model both the Big Bang and the later occurring rapid inflation of the Universe. The gravitational interaction between real galaxies takes time to propagate. The effect of the propagation delay of the gravitational interaction between galaxies involves the current position of one galaxy and the past and estimated future position of another galaxy. The first galaxy has information about the past position and velocity of the other galaxy and can use this information to estimate the future position of that other galaxy. Therefore, the process is causal.

For small delays, the position differences divided by the square of the round-trip delay time, can be approximated by the second derivative of the distances between galaxies. Since the distances between galaxies are proportional to the radius of the sphere in the Universe, one can approximate the second derivative of the distances between galaxies by the second derivative of the radius of the sphere in the Universe multiplied by an adjustable parameter. The Einstein equation is an equation for the second derivative of the radius of the sphere in the Universe. By adding the delay term to Einstein's equation, one now has two second derivatives of the radius of the sphere in the Universe. The two second derivative terms have opposite signs. When the two are equal, each term must be very large since this difference is equal to a finite term. Thus, the equation for the second derivative or acceleration of the radius of the sphere in the Universe has a singularity. When the resulting equation is integrated twice to calculate the radius, the singularity results in a step in the expansion of the radius, the inflation of the Universe.

\section{Acknowledgements}

I thank my wife Marlene Danzig Kornreich for her suggestions to the content, analysis and for making the text more understandable to a reader. I also thank her for proofreading and working together on the manuscript. 


\section{Conflicts of Interest}

The author declares no conflicts of interest regarding the publication of this paper.

\section{References}

[1] Einstein, A., Lorentz, H.A., Weyl, H. and Minkowsk, H. (1952) The Principles of Relativity. Dover Publishing Co, Toronto, ON.

[2] Weaver, D. and Villard, R. (2018) Measuring Universe Expansion Reveals Mystery-Is Something Unpredicted Going on in the Depths of Space? EarthSky Voices in Space.

[3] Baez, J., Bunn, C. and Emory, F. (2005) The Meaning of Einstein's Equation. American Journal of Physics, 73, 644. math.ucr.edu/home/baez/einstein.pd https://doi.org/10.1119/1.1852541

[4] Tedrode, H.M. (1922) Über den Wirkungszusammenhang der Welt. Eine Erweiterung der Klassischen Dynamik. Zeitschrift für Physik, 10, 317-328. https://doi.org/10.1007/BF01332574

[5] Wheeler, J.A. and Feynman, R. (1945) Absorber Theory and the Radiation Arrow of Time. Review of Modern Physics, 17, 157-181. https://doi.org/10.1103/RevModPhys.17.157

[6] Dirac, P.A.M. (1938) Classical Theory of Radiating Electrons. Proceedings of the Royal Society A, 167, 148. https://doi.org/10.1098/rspa.1938.0124

[7] Lande, A. (1950) On Advanced and Retarded Potentials. Letter to Editor. Phys. Rev., 80, 283.

[8] Kornreich, P. (2017) Reaction Mechanics of Point Objects. Journal of Applied Mathematics and Physics, 5, 137-152. https://doi.org/10.4236/jamp.2017.51015

[9] Stephen Hawking Center for Theoretical Cosmology. The Origins of the Universe: Inflation. http://www.ctc.cam.ac.uk/outreach/origins/inflation_zero.php

[10] Peebles, P.J.E. (1984) Tests of Cosmological Models Constrained by Inflation. Astrophysical Journal, 284, 439-444. arxiv.org gr-qc

[11] Ruben, D. and Hayden, B. (2016) Is the Expansion of the Universe Accelerating? All Signs Point to Yes. arXiv 1610.08972v3 